\title{
UNIFORM CONTINUITY OF QUASICONFORMAL MAPPINGS ONTO GENERALIZED JOHN DOMAINS
}

\author{
Chang-Yu Guo \\ University of Jyväskylä, Department of Mathematics and Statistics \\ P. O. Box 35, FI-40014 University of Jyväskylä, Finland; changyu.c.guo@jyu.fi
}

\begin{abstract}
We study uniform continuity of quasiconformal mappings onto $\delta$-Gromov-hyperbolic $\varphi$-John domains. The general $\varphi$-John case is also investigated.
\end{abstract}

\section{Introduction}

Gehring and Martio [4] have shown that if $\Omega^{\prime} \subset \mathbf{R}^{n}$ is a uniform domain and $\Omega \subset \mathbf{R}^{n}$ is a John domain, then each quasiconformal mapping $f: \Omega^{\prime} \rightarrow \Omega$ is (globally) Hölder continuous. Later, Koskela, Onninen and Tyson [20] enhanced the result by removing the uniformity condition on the source domain $\Omega^{\prime}$, i.e. if $\Omega^{\prime} \subset \mathbf{R}^{n}$ is a bounded domain and if $\Omega \subset \mathbf{R}^{n}$ is a John domain, then each quasiconformal mapping $f: \Omega^{\prime} \rightarrow \Omega$ is (globally) Hölder continuous if $\Omega^{\prime}$ is equipped with the internal metric.

Recall that a bounded domain $\Omega \subset \mathbf{R}^{n}$ is a John domain if there is a constant $C$ and a point $x_{0} \in \Omega$ so that, for each $x \in \Omega$, one can find a rectifiable curve $\gamma:[0,1] \rightarrow \Omega$ with $\gamma(0)=x, \gamma(1)=x_{0}$ and with

$$
C d(\gamma(t), \partial \Omega) \geq l(\gamma([0, t]))
$$

for each $0<t \leq 1$. Alternatively, one may replace the right-hand side with $\mid \gamma(t)-$ $x_{0} \mid$ or with $\operatorname{diam}(\gamma([0, t]))$, see $[22]$ and rectifiability of $\gamma$ is not needed in these two modifications to the above definition. Here John refers to F. John who used this condition in his work on elasticity [18]; Martio and Sarvas [21] introduced this terminology. The class of John domains includes all smooth domains, Lipschitz domains and certain fractal domains (for example the snowflake domain).

Motivated by the recent studies on generalized quasidisks [8, 11], Guo and Koskela [9] have introduced the class of $\varphi$-John domains, which form a natural generalization of John domains. Let $\varphi$ be a continuous, increasing function with $\varphi(0)=0$ and $\varphi(t) \geq t$ for all $t>0$. A bounded domain $\Omega$ is termed a $\varphi$-length John domain when (1.1) is replaced with

$$
\varphi(C d(\gamma(t), \partial \Omega)) \geq l(\gamma([0, t])) .
$$

The concepts of $\varphi$-dist and $\varphi$-diam John domains are defined analogously. A corresponding curve $\gamma$ is called a $\varphi$-dist (diam, length) John curve. In order to avoid complicated formulations for our results, we assume in what follows that $\varphi^{-1}$ is doubling and that $t \mapsto \frac{t}{\varphi^{-1}(t)}$ is decreasing. Here doubling requires that $\varphi^{-1}(2 t) \leq C \varphi^{-1}(t)$ for some constant $C$ and all $t>0$. Our second condition is in practise harmless, but the

doi:10.5186/aasfm.2015.4010

2010 Mathematics Subject Classification: Primary 30C62,30C65.

Key words: John domain, inner uniform domain, $\delta$-Gromov-hyperbolic domain, quasiconformal mapping, quasihyperbolic geodesic.

The author was partially supported by the Academy of Finland grant 131477. 
doubling condition rules out the case of exponential exterior cusps. For $\varphi(t)=t^{1 / s}$, $s>1, \varphi$-length John domains are customarily called $s$-John domains [24].

To read our first main result below, recall that the internal metric $d_{I}$ on a domain $\Omega \subset \mathbf{R}^{n}$ is defined by taking the infimum of the lengths over all rectifiable curves in $\Omega$ joining the desired pair of points. Observe that always

$$
\left|f\left(x^{\prime}\right)-f\left(y^{\prime}\right)\right| \leq d_{I}\left(f\left(x^{\prime}\right), f\left(y^{\prime}\right)\right) .
$$

Theorem 1.1. Let $\Omega^{\prime} \subset \mathbf{R}^{n}$ be a bounded domain and let $\Omega \subset \mathbf{R}^{n}$ be a domain that is Gromov hyperbolic in the quasihyperbolic metric. Let $f: \Omega^{\prime} \rightarrow \Omega$ be a $K$ quasiconformal mapping.

(1) If $\Omega$ is $\varphi$-length John, then $f$ is uniformly continuous with a modulus of continuity of the form

$$
d_{I}\left(f\left(x^{\prime}\right), f\left(y^{\prime}\right)\right) \leq C \psi^{-1}\left(C \log \left(M / d_{I}\left(x^{\prime}, y^{\prime}\right)\right)\right)
$$

where

$$
\psi(t)=\int_{t}^{M} \frac{d s}{\varphi^{-1}(s)} .
$$

(2) If $\Omega$ is $\varphi$-dist (diam) John and is bounded in the internal metric, then $f$ is uniformly continuous with a modulus of continuity of the form

$$
d_{I}\left(f\left(x^{\prime}\right), f\left(y^{\prime}\right)\right) \leq C \psi^{-1}\left(C \log \left(M / d_{I}\left(x^{\prime}, y^{\prime}\right)\right)\right)
$$

where

$$
\psi(t)=\int_{t}^{M} \frac{s^{n-1} d s}{\left(\varphi^{-1}(s)\right)^{n}} .
$$

For a version of Theorem 1.1 in terms of growth conditions on the quasihyperbolic metric, see [16]. Theorem 1.1 is not covered by those results (see e.g. [7, pp. 10-11]) and the observation that one can use the internal metric on the left-hand side in the result in [16] goes back to Koskela and Nieminen [19].

The proof of Theorem 1.1 is based on a systematic study of $\varphi$-dist (diam, length) John domains. In particular, it relies crucially on the fact established below that the quasihyperbolic geodesics in a $\delta$-Gromov-hyperbolic $\varphi$-John domain are $\varphi$-inner uniform curves; see Section 3.1 below for the definition of $\delta$-Gromov-hyperbolic domains. In particular, the quasihyperbolic geodesics starting from the center in a $\delta$-Gromov-hyperbolic $\varphi$-John domain are $\varphi$-John curves. Since each domain that is quasiconformally equivalent to a uniform domain is necessarily $\delta$-Gromov hyperbolic in the quasihyperbolic metric, this conclusion is of interest. Another important ingredient is the use of geodesics towards bounds on the conformal capacity, see [14, 17, 20] for more on this idea. The sharpness of the modulus estimates in Theorem 1.1 is demonstrated in [9] in the planar case.

On the other hand, Theorem 1.1 does not hold if one drops the Gromov hyperbolicity assumption. In the model $s$-John case, by using an idea from [15], we obtain our second main result. 
Theorem 1.2. Let $\Omega^{\prime} \subset \mathbf{R}^{n}$ be a domain and $\Omega$ be an $s$-John domain with $s \in\left(1,1+\frac{1}{n-1}\right)$. Then each quasiconformal mapping $f: \Omega^{\prime} \rightarrow \Omega$ satisfies

$$
D_{I}\left(f\left(x^{\prime}\right), f\left(y^{\prime}\right)\right) \leq C\left(\log \frac{1}{C d_{I}\left(x^{\prime}, y^{\prime}\right)}\right)^{-\frac{1}{s-1}}
$$

for every pair $x^{\prime}, y^{\prime}$ of distinct points in $\Omega^{\prime}$, where $D_{I}$ is defined by taking the infimum of the diameters over all rectifiable curves in $\Omega$ joining the desired pair of points.

The result in Theorem 1.2 is essentially sharp as the following two examples indicates.

Example 1.3. [10, Theorem 1.2] There exist a bounded domain $\Omega^{\prime} \subset \mathbf{R}^{2}$, a 2-John domain $\Omega \subset \mathbf{R}^{2}$, and a quasiconformal mapping $f: \Omega^{\prime} \rightarrow \Omega$ such that $f$ is not uniformly continuous with respect to the metrics $d(x, y)=|x-y|$ in $\Omega$ and $d_{I}$ in $\Omega^{\prime}$.

Example 1.4. [10, Theorem 1.4] Let $n \geq 3$. There exist a bounded domain $\Omega^{\prime} \subset$ $\mathbf{R}^{n}$, a domain $\Omega \subset \mathbf{R}^{n}$ that is $s$-John for any $s \in\left(1+\frac{1}{n-1}, \infty\right)$, and a quasiconformal mapping $f: \Omega^{\prime} \rightarrow \Omega$ such that $f$ is not uniformly continuous with respect to the metrics $d(x, y)=|x-y|$ in $\Omega$ and $d_{I}$ in $\Omega^{\prime}$.

Theorem 1.2 is covered by a more general result, namely Theorem 6.1 below, that deals with more general $\varphi$-John domains. However, both the formulation and the proof of Theorem 6.1 are more complicated.

This paper is organized as follows. Section 2 contains notation and the basic definitions. In Section 3, we characterize $\delta$-Gromov-hyperbolic $\varphi$-John domains. Section 4 and 5 contain some auxiliary results. We prove a more general version of Theorem 1.2 in Section 6. Finally, in Section 7, we prove our main results.

\section{Notation and definitions}

The closure of a set $U \subset \mathbf{R}^{n}$ is denoted $\bar{U}$ and the boundary $\partial U$. The open ball of radius $r>0$ centered at $x \in \mathbf{R}^{n}$ is denoted by $B(x, r)$ and in the case of the unit ball we omit the centre and the radius, writing $B:=B(0,1)$. In the planar case, we write $\mathbf{D}$ for the unit disk. The boundary of $B(x, r)$ will be denoted by $S(x, r)$ and in the case of the boundary of the unit ball, writing $S:=S(0,1)$. The symbol $\Omega$ always refers to a domain, i.e. a connected and open subset of $\mathbf{R}^{n}$.

A domain bounded by a Jordan curve will be named Jordan domain. Recall that a set $E$ is called linearly locally connected (LLC) if there is a constant $C \geq 1$ so that

- (LLC-1) each pair of points in $B(x, r) \cap E$ can be joined by an $\operatorname{arc}$ in $B(x, C r) \cap$ $E$, and

- (LLC-2) each pair of points in $E \backslash B(x, C r)$ can be joined by an arc in $E \backslash$ $B(x, r)$.

We need a weaker version of this condition, which was introduced in [11]. We say that $\Omega$ is $(\varphi, \psi)$-locally connected $((\varphi, \psi)$-LC) if

- $(\varphi$-LC-1) each pair of points in $B(x, r) \cap \Omega$ can be joined by an arc in $B(x, \varphi(r)) \cap \Omega$, and

- $(\psi$-LC-2) each pair of points in $\Omega \backslash B(x, r)$ can be joined by an arc in $\Omega \backslash$ $B(x, \psi(r))$, 
where $\varphi, \psi:[0, \infty) \rightarrow[0, \infty)$ are smooth increasing functions such that $\varphi(0)=\psi(0)=$ $0, \varphi(r) \geq r$ and $\psi(r) \leq r$ for all $r>0$.

We say that $\Omega$ is $\varphi$-dist(diam, length) inner uniform, if there exists a constant $C>0$ such that each pair of points $x_{1}, x_{2} \in \Omega$ can be joined by a curve $\gamma$ in $\Omega$ for which

$$
\varphi(C d(\gamma(t), \partial \Omega)) \geq \min _{i=1,2} S\left(\gamma\left(\left[x_{j}, \gamma(t)\right]\right)\right)
$$

and

$$
d_{I}\left(x_{1}, x_{2}\right) \geq C S(\gamma)
$$

with $S(\gamma)$ equal to $|\gamma(1)-\gamma(0)|, \operatorname{diam}(\gamma)$ and $l(\gamma)$, respectively. If $\varphi(t)=t$, we recover the definition of an inner uniform domain.

Next, recall that the quasihyperbolic metric $k_{\Omega}$ in a domain $\Omega \subsetneq \mathbf{R}^{n}$ is defined to be

$$
k_{\Omega}(x, y)=\inf _{\gamma} k_{\Omega^{-}} \operatorname{length}(\gamma)
$$

where the infimum is taken over all rectifiable curves $\gamma$ in $\Omega$ which join $x$ to $y$ and

$$
k_{\Omega^{-}} \text {length }(\gamma)=\int_{\gamma} \frac{d s}{d(x, \partial \Omega)}
$$

denotes the quasihyperbolic length of $\gamma$ in $\Omega$. This metric was introduced by Gehring and Palka in [6]. A curve $\gamma$ joining $x$ to $y$ for which $k_{\Omega}$-length $(\gamma)=k_{\Omega}(x, y)$ is called a quasihyperbolic geodesic. Quasihyperbolic geodesics joining any two points of a proper subdomain of $\mathbf{R}^{n}$ always exists; see [5, Lemma 1]. Given two points $x, y \in \Omega$, we denote by $[x, y]$ the quasihyperbolic geodesic that joins $x$ and $y$.

For an increasing function $\tau:[0, \infty) \rightarrow[0, \infty)$ with $\tau(0)=0$, we denote by $\mathcal{H}_{\tau}^{\infty}$ the Hausdorff $\tau$-content: $\mathcal{H}_{\tau}^{\infty}(E)=\inf \sum_{i} \tau\left(r_{i}\right)$, where the infimum is taken over all coverings of $E \subset \mathbf{R}^{n}$ with balls $B\left(x_{i}, r_{i}\right), i=1,2, \ldots$ When $\tau(t)=t^{s}$ for some $0<s<\infty$, we write $\mathcal{H}_{s}^{\infty}=\mathcal{H}_{\tau}^{\infty}$.

For disjoint compact sets $E$ and $F$ in the domain $\Omega$, we denote by $\operatorname{Cap}(E, F, \Omega)$ the conformal $n$-capacity of the pair $(E, F)$ :

$$
\operatorname{Cap}(E, F, \Omega)=\inf _{u} \int_{\Omega}|\nabla u(x)|^{n} d x
$$

where the infimum is taken over all continuous functions $u \in W_{l o c}^{1, n}(\Omega)$ which satisfy $u(x) \leq 0$ for $x \in E$ and $u(x) \geq 1$ for $x \in F$.

For $K \geq 1$, a homeomorphism $f: \Omega^{\prime} \rightarrow \Omega$ is said to be $K$-quasiconformal if

$$
\frac{1}{K} \operatorname{Cap}\left(E, F, \Omega^{\prime}\right) \leq \operatorname{Cap}(f(E), f(F), \Omega) \leq K \operatorname{Cap}\left(E, F, \Omega^{\prime}\right)
$$

for all disjoint compact sets $E, F \subset \Omega^{\prime}$.

Let $\Omega$ be a bounded domain in $\mathbf{R}^{n}, n \geq 2$. Then $\mathbf{W}=\mathbf{W}(\Omega)$ denotes a Whitney decomposition of $\Omega$, i.e. a collection of closed cubes $Q \subset \Omega$ with pairwise disjoint interiors and having edges parallel to the coordinate axes, such that $\Omega=\cup_{Q \in \mathbf{W}} Q$. Also, the diameters of $Q \in \mathbf{W}$ are in the set $\left\{2^{-j}: j \in \mathbf{Z}\right\}$ and satisfy the condition

$$
\operatorname{diam}(Q) \leq \operatorname{dist}(Q, \partial \Omega) \leq 4 \operatorname{diam}(Q) .
$$

For $j \in \mathbf{Z}$ we define

$$
\mathbf{W}_{j}=\left\{Q \in \mathbf{W}: \operatorname{diam}(Q)=2^{-j}\right\}
$$


Note that when we write $f(x) \lesssim g(x)$, we mean that $f(x) \leq C g(x)$ is satisfied for all $x$ with some fixed constant $C \geq 1$. Similarly, the expression $f(x) \gtrsim g(x)$ means that $f(x) \geq C^{-1} g(x)$ is satisfied for all $x$ with some fixed constant $C \geq 1$. We write $f(x) \approx g(x)$ whenever $f(x) \lesssim g(x)$ and $f(x) \gtrsim g(x)$.

\section{Characterization of $\delta$-Gromov-hyperbolic $\varphi$-John domains}

In this section, we study the relation between $\varphi$-John domains and $\varphi$-inner uniform domains. The aim is to show the following two results.

Theorem 3.1. Let $\Omega \subset \mathbf{R}^{n}$ be a bounded domain that is $\delta$-Gromov-hyperbolic in the quasihyperbolic metric. Then the following statements hold:

(1) $\Omega$ is $\varphi$-dist John if and only if it is $\varphi$-diam John.

(2) If $\Omega$ is $\varphi$-diam John, then $\Omega$ is $\eta$-length John for

$$
\eta(t)=C \int_{0}^{\varphi(C t)}\left(\frac{s}{\varphi^{-1}(s)}\right)^{n-1} d s,
$$

provided this integral converges.

The statement (2) is essentially sharp in the sense that $\eta$ defined in (3.1) is best possible.

Theorem 3.2. Let $\Omega \subset \mathbf{R}^{n}$ be a bounded domain that is $\delta$-Gromov-hyperbolic in the quasihyperbolic metric. Then the following statements are equivalent:

(1) $\Omega$ is $\varphi$-dist (diam, length) John;

(2) $\Omega$ is $\varphi$-dist (diam, length) inner uniform.

Theorem 3.1 and Theorem 3.2 can be regarded as higher-dimensional generalizations of the corresponding results in [9].

3.1. $\delta$-Gromov-hyperbolic domains. Following [2], we say that a domain $\Omega \subset \mathbf{R}^{n}$ is $\delta$-Gromov-hyperbolic (in the quasihyperbolic metric), $\delta \geq 0$, if for all triples of quasihyperbolic geodesics $[x, y],[y, z],[z, x]$ in $\Omega$ every point in $[x, y]$ is within distance $\delta$ from $[y, z] \cup[z, x]$ in the quasihyperbolic metric. The property is often expressed by saying that geodesic triangles in $\Omega$ are $\delta$-thin.

It is well-known that if $\Omega \subset \mathbf{R}^{n}$ is $\delta$-Gromov-hyperbolic, then the GehringHayman inequality holds in $\Omega$. More precisely, there exists a constant $C \geq 1$ such that for every curve $\gamma$ in $\Omega$ with end points $x$ and $y$ we have that

$$
l([x, y]) \leq C l(\gamma) .
$$

Moreover, the following separation property holds: there exists a constant $C \geq 1$ such that for each pair of points $x, y \in \Omega$, for each $z \in[x, y]$, and for every curve $\gamma$ in $\Omega$ joining $x$ to $y$ it holds that

$$
B^{I}(z, C d(z, \partial \Omega)) \cap \gamma \neq \emptyset .
$$

Above, $B^{I}(z, s)$ denotes the internal ball of radius $s$, i.e. set of points in $\Omega$ with internal distance less than $s$ from $z$. In particular,

$$
B(z, C d(z, \partial \Omega)) \cap \gamma \neq \emptyset .
$$

See [2] for the above mentioned facts. 
3.2. Relation of $\varphi$-John domain and $\varphi$-inner uniform domain. Recall that we have assumed in the introduction that the gauge function $\varphi$ satisfies that $\varphi(t) \geq t$ and $\varphi^{-1}$ is doubling.

Lemma 3.3. [9, Lemma 3.5] Let $C_{1} \geq 1, C_{2} \geq 1$, and $C_{3} \geq 1$ be given. There exists a constant $C$, depending only on $C_{0}, C_{1}, C_{2}$ and $C_{3}$, such that

$$
C_{1} \varphi\left(C_{2} t\right)+C_{3} t \leq \varphi(C t)
$$

for all $t>0$. Above, $C_{0}$ is the doubling constant of $\varphi^{-1}$.

The following result generalizes the well-known fact that bounded inner uniform domains are John.

Lemma 3.4. Let $\Omega \subset \mathbf{R}^{n}$ be a bounded domain. Then $\Omega$ is $\varphi$-dist (diam, length) John if and only if each pair of points can be joined by a curve satisfying the $\varphi$-dist (diam, length) inner uniformity condition (2.1). In particular, bounded $\varphi$-dist (diam, length) inner uniform domains are $\varphi$-dist (diam, length) John.

Proof. We only prove the length case, since the other cases can be proved similarly. Let us suppose first that $\Omega$ is $\varphi$-length John with center $x_{0}$. Let $a_{1}, a_{2} \in \Omega$. We may connect each $a_{i}$ to $x_{0}$ by a $\varphi$-length John curve $\gamma_{i}, i=1,2$. The desired curve $\gamma$ that satisfies the $\varphi$-length inner uniformity condition (2.1) can be chosen as the union of $\gamma_{i}$ 's in the sense that $\gamma\left(a_{1}, x_{0}\right)=\gamma_{1}\left(a_{1}, x_{0}\right)$ and $\gamma\left(x_{0}, a_{2}\right)=\gamma\left(x_{0}, a_{2}\right)$.

The proof of the reverse direction can be found in [9, Proof of Lemma 4.3]. Note that the refered proof above only deals with the case $n=2$, but it carries through in arbitrary dimensions.

Next, we show that a bounded $\varphi$-John domain is necessarily $\varphi$-LC-2.

Lemma 3.5. Let $\Omega \subset \mathbf{R}^{n}$ be a bounded $\varphi$-dist John domain. Then $\Omega$ is $\varphi-L C-2$.

Proof. The proof is essentially contained in [9, Proof of Theorem 1.1(1)]. One only needs to use Lemma 3.4 to replace the corresponding Lemma 4.2 used in [9, Proof of Theorem 1.1, part 1] and observe that the dimension $n=2$ plays no role in the proof.

Lemma 3.6. Let $\Omega \subset \mathbf{R}^{n}$ be a bounded $\varphi$-length John domain. Then $\Omega$ is $\varphi$-LC-2 with respect to the internal metric, i.e. for all $z \in \Omega, r>0$, any two points in $\Omega \backslash B^{I}(z, \varphi(C r))$ can be joined in $\Omega \backslash B^{I}(z, r)$.

Proof. By Lemma 3.3, we only need to show that for any $z \in \Omega$ and $r>0$, at most one component of $\Omega \backslash \overline{B^{I}}(z, r)$ meets $\Omega \backslash \overline{B^{I}}(z, 3 \varphi(2 C r))$. Towards this end, let $a_{1}$ and $a_{2}$ be two points in $\Omega \backslash \overline{B^{I}}(z, 3 \varphi(2 C r))$ which belongs to different components of $\Omega \backslash \overline{B^{I}}(z, r)$. Lemma 3.4 implies that we may find a curve $\gamma$ joining $a_{1}$ and $a_{2}$ such that for any $t \in(0,1)$,

$$
\min \{l(\gamma([0, t])), l(\gamma([t, 1]))\} \leq \varphi(C d(\gamma(t), \partial \Omega)) .
$$

Let $y \in \gamma \cap \overline{B^{I}}(z, r)$. Since $d_{I}\left(y, a_{i}\right) \geq 3 \varphi(2 C r)-r$, the ball

$$
B\left(y, \frac{\varphi^{-1}(3 \varphi(2 C r)-r)}{C}\right)
$$


lies in $\Omega$. Since $\bar{B}(z, r)$ cannot be contained in $\Omega$, this implies that

$$
\frac{\varphi^{-1}(3 \varphi(2 C r)-r)}{C} \leq 2 r
$$

or equivalently

$$
3 \varphi(2 C r)-r \leq \varphi(2 C r),
$$

which contradicts the assumption that $\varphi(t) \geq t$.

Note that one cannot conclude from Lemma 3.4 that $\Omega$ is $\varphi$-diam (length) inner uniform whenever it is $\varphi$-diam (length) John, since the diameter (length) of the curve from Lemma 3.4 that satisfies the $\varphi$-diam (length) inner uniformity condition (2.1) is not necessarily bounded from above by a (universal) constant times the internal distance. In fact, if $E$ is any compact subset of the line segment $\left[0, e_{1}\right] \subset \mathbf{R}^{2}$, then $\mathbf{D} \backslash E$ is John, but it is not necessarily inner uniform. On the other hand, this is the case if $\Omega$ is additionally assumed to be $\delta$-Gromov-hyperbolic.

Proposition 3.7. Let $\Omega \subset \mathbf{R}^{n}$ be a bounded $\delta$-Gromov-hyperbolic $\varphi$-dist (length) John domain. Then quasihyperbolic geodesics in $\Omega$ are $\varphi$-diam (length) inner uniform curves. In particular, $\Omega$ is $\varphi$-diam (length) inner uniform.

Proof. First assume that $\Omega$ is $\varphi$-dist (length) John and fix $x, y \in \Omega$. We want to show that the quasihyperbolic geodesic $[x, y]$ in $\Omega$ is the desired $\varphi$-diam inner uniform curve. Note first that by the Gehring-Hayman inequality (3.2) we only need to verify the inner uniformity condition (2.1) for $[x, y]$.

To this end, let $z \in[x, y]$. Note that Lemma 3.5 implies that $\Omega$ is $\varphi$-LC-2, i.e. every two points in $\Omega \backslash B(z, \varphi(C d(z, \partial \Omega)))$ can be joined in $\Omega \backslash B\left(z, \varphi^{-1}\left(C_{1}^{-1} \varphi(C d(z\right.\right.$, $\partial \Omega()))$ ). On the other hand, the separation condition for $[x, y]$ implies that there exists a constant $C^{\prime}$ such that for every curve $\gamma$ in $\Omega$ joining $x$ to $y$ it holds that

$$
B\left(z, C^{\prime} d(z, \partial \Omega)\right) \cap \gamma \neq \emptyset .
$$

We claim that either $x$ or $y$ must be in $B(z, \varphi(C d(z, \partial \Omega))) \cap \Omega$, where $C$ is chosen, depending only on $C_{1}, C^{\prime}$ and the doubling coefficient of $\varphi$, such that $\varphi(C d(z, \partial \Omega)) \geq$ $C_{1} \varphi\left(C^{\prime} d(z, \partial \Omega)\right)$. If not, the $\varphi$-LC-2 condition would imply that $x$ and $y$ can be joined in $\Omega$ outside the $B\left(z, \varphi^{-1}\left(C_{1}^{-1} \varphi(C d(z, \partial \Omega))\right)\right)$, which contradicts (3.6). This in return implies that $[x, y]$ satisfies $(2.1)$.

The proof of the $\varphi$-length John case can be proceeded similarly and one only needs to use the fact that $\Omega$ is $\varphi$-LC-2 with respect to the internal metric, which is provided by Lemma 3.6.

Proposition 3.8. Let $\Omega \subset \mathbf{R}^{n}$ be a bounded $\delta$-Gromov-hyperbolic $\varphi$-dist (diam, length) John domain. Then quasihyperbolic geodesics starting from the center are $\varphi$-dist (diam, length) John curves in $\Omega$.

Proof. We only prove the dist case since the other two cases can be proved similarly. Let $\lambda=\frac{\operatorname{diam} \Omega}{2}$. Then there exists a constant $C>0$, depending only on $x_{0}$, $\Omega$ and $\varphi$, such that $B\left(x_{0}, \frac{\varphi^{-1}(\lambda / 2)}{C}\right) \subset \Omega$. On the other hand, since $\Omega$ is $\varphi$-dist John, $\operatorname{diam} \Omega \leq 2 \varphi(C \lambda)$. Let $y \in \Omega$. By Proposition 3.7, $\left[y, x_{0}\right]$ is a $\varphi$-dist inner uniform curve. Let $x \in\left[y, x_{0}\right]$. We may assume that $|x-y|>\left|x-x_{0}\right|$. If $\left|x-x_{0}\right|<\frac{\varphi^{-1}(\lambda / 2)}{2 C}$, 
then $d(x, \partial \Omega) \geq \frac{\varphi^{-1}(\lambda / 2)}{2 C}$. It follows that

$$
\varphi(2 C d(x, \partial \Omega)) \geq \lambda / 2 \geq \frac{|x-y|}{4} .
$$

In the other case, $\left|x-x_{0}\right| \geq \frac{\varphi^{-1}(\lambda / 2)}{2 C}$. Then the inner uniformity gives that

$$
\varphi(C d(x, \partial \Omega)) \geq\left|x-x_{0}\right| \geq \frac{\varphi^{-1}(\lambda / 2)}{2 C} \geq \frac{\varphi^{-1}(\lambda / 2)}{4 C \lambda}|x-y| .
$$

The claim follows from Lemma 3.3.

Proposition 3.7 can be proved without appealing to Lemma 3.5 if we know that quasihyperbolic geodesics starting from the center are $\varphi$-John curves in $\Omega$.

Lemma 3.9. Let $\Omega \subset \mathbf{R}^{n}$ be a bounded $\delta$-Gromov-hyperbolic $\varphi$-dist (diam, length) John domain. If quasihyperbolic geodesics starting from the center are $\varphi$ dist (diam, length) John curves in $\Omega$, then quasihyperbolic geodesics joining any two points in $\Omega$ are $\varphi$-dist (diam, length) inner uniform curves.

Proof. We only consider the length case since the proofs for the other cases are similar. Let $x, y \in \Omega$. We want to show that $[x, y]$ is the desired $\varphi$-length inner uniform curve.

To this end, let us fix a point $\omega \in[x, y]$ with $\omega \neq x, y$. Then $\left[x, x_{0}\right],\left[y, x_{0}\right]$ and $[x, y]$ form a quasihyperbolic triangle in $\Omega$ and hence there exists a point $z \in$ $\left[x, x_{0}\right] \cup\left[y, x_{0}\right]$ such that

$$
k_{\Omega}(\omega, z) \leq \delta<\infty .
$$

As a consequence, there exists a constant $M>0$, depending only on $\delta$, such that

$$
d_{I}(\omega, z) \leq M \min \{d(\omega, \partial \Omega), d(z, \partial \Omega)\}
$$

and $d(\omega, \partial \Omega) \geq \frac{1}{M} d(z, \partial \Omega)$. If $l([y, z]) \gtrsim l([\omega, z])$, then the length-Gehring-Hayman implies that

$$
l([\omega, y]) \lesssim l([\omega, z])+l([y, z]) \lesssim l([y, z]) \leq \varphi(d(z, \partial \Omega)) \leq \varphi(M d(x, \partial \Omega)) .
$$

If $l([y, z]) \lesssim l([\omega, z])$, then the length-Gehring-Hayman implies that

$$
l([\omega, y]) \lesssim l([\omega, z])+l([y, z]) \lesssim l([\omega, z]) \lesssim d_{I}(\omega, z) \lesssim d(\omega, \partial \Omega) .
$$

Remark 3.10. Proposition 3.7, Proposition 3.8 and Lemma 3.9 give a short positive answer to Heinonen's question [13, Question 2], previously solved in [2].

We next show that in a bounded $\delta$-Gromov-hyperbolic $\varphi$-length John domain, the length of a quasihyperbolic geodesic can be controlled by its diameter.

Proposition 3.11. Let $\Omega \subset \mathbf{R}^{n}$ be a bounded $\delta$-Gromov-hyperbolic $\varphi$-length John domain. Then

$$
l(\gamma) \leq \varphi(C \operatorname{diam}(\gamma))
$$

for any quasihyperbolic geodesic $\gamma$ in $\Omega$, where $C$ is a constant depending only on the associated data.

Proof. Let $\gamma=[x, y] \subset \Omega$ be a quasihyperbolic geodesic joining $x$ to $y$. Choose $z \in \gamma$ such that

$$
l([x, z])=l([z, y]) .
$$


By Proposition 3.7, $\gamma$ is a $\varphi$-length inner uniform curve and hence

$$
\min \{l([x, u], l([u, y])\} \leq \varphi(C d(u, \partial \Omega))
$$

for any $u \in \gamma$. We consider two cases.

Case 1: $\max \{|x-z|,|y-z|\} \geq d(z, \partial \Omega)$. Without loss of generality, we may assume that $|x-z| \geq d(z, \partial \Omega)$. Since $\operatorname{diam}([x, z]) \geq|x-z|$, we easily obtain that

$$
\varphi(C \operatorname{diam}([x, z])) \geq \varphi(C d(z, \partial \Omega)) \geq l([x, z]) .
$$

Therefore,

$$
l(\gamma)=2 l([x, z]) \leq 2 \varphi(C \operatorname{diam}(\gamma)) .
$$

Case 2: $\max \{|x-z|,|y-z|\}<d(z, \partial \Omega)$. In this case, $x, y \in B(z, d(z, \partial \Omega))$ and hence the line segments $\overline{x z}$ and $\overline{z y}$ are contained in $\Omega$. The Gehring-Hayman inequality (3.2) implies that

$$
l([x, z]) \leq C|x-z|
$$

Therefore,

$$
l(\gamma)=2 l([x, z]) \leq C \operatorname{diam}(\gamma) .
$$

The claim then follows from Lemma 3.3.

\subsection{Proofs of Theorem 3.1 and Theorem 3.2}

Proof of Theorem 3.1. Part (1) follows directly from Proposition 3.7 and Lemma 3.4. Part (2) follows by an obvious modification to [9, Proof of Theorem 1.1, part 2].

Proof of Theorem 3.2. The claim is a direct consequence of Lemma 3.4 and Proposition 3.7.

\section{Estimates for the number of Whitney cubes intersecting a $\varphi$-John shadow}

Let $\Omega \subset \mathbf{R}^{n}$ be a $\varphi$-John domain with center $x_{0}$. Let $\mathbf{W}=\mathbf{W}(\Omega)$ be a Whitney decomposition of $\Omega$. Fix a set $E \subset \Omega$. When $\omega \in E$, we fix a $\varphi$-John curve $\mathbf{J}_{\varphi}\left(\omega, x_{0}\right)$ joining $\omega$ to $x_{0}$ in $\Omega$. We then define

$$
P(\omega)=\left\{Q \in \mathbf{W}: Q \cap \mathbf{J}_{\varphi}\left(\omega, x_{0}\right) \neq \emptyset\right\} .
$$

Write

$$
P(E)=\bigcup_{\omega \in E} P(\omega)
$$

The $\varphi$-John shadow $S_{E}(Q)$ of a cube $Q \in \mathbf{W}$ on the set $E$ is now defined by

$$
S_{E}(Q)=\{\omega \in E: Q \in P(\omega)\} .
$$

Lemma 4.1. Let $\Omega \subset \mathbf{R}^{n}$ be a $\varphi$-dist John domain, and let $\mathbf{W}$ be a Whitney decomposition of $\Omega$. Then

$$
\operatorname{diam}\left(S_{E}(Q)\right) \leq 2 \varphi(5 C \operatorname{diam}(Q))
$$

for each $Q \in \mathbf{W}$. 
Proof. Let $\omega \in S(Q)$. We may find a $\varphi$-dist John curve $\gamma$ joining $\omega$ to $x_{0}$ in $\Omega$ so that $\gamma\left(t_{Q}\right) \in Q$ for some $t_{Q} \in[0, l(\gamma)]$. It follows that

$$
d(\omega, Q) \leq d\left(\omega, \gamma\left(t_{Q}\right)\right) \leq \varphi\left(C d\left(\gamma\left(t_{Q}\right), \partial \Omega\right)\right) \leq \varphi(5 C \operatorname{diam}(Q)) .
$$

The claim follows immediately from (4.2) and the triangle inequality.

Lemma 4.2. Let $\Omega \subset \mathbf{R}^{n}$ be a $\varphi$-dist John domain, and let $\mathbf{W}$ be a Whitney decomposition of $\Omega$. Let $E \subset \Omega$. Then there exists a positive constant $C$, depending only on $n$, such that for each $j \in \mathbf{Z}$ and $\omega \in E$,

$$
\#\left\{Q \in \mathbf{W}_{j}: \omega \in S_{E}(Q)\right\} \leq C\left(\frac{\varphi\left(6 C \cdot 2^{-j}\right)}{2^{-j}}\right)^{n} .
$$

Proof. If $\omega \in S_{E}(Q)$, then $Q \in P(\omega)$. By (4.2), we obtain that

$$
Q \subset B(\omega, \varphi(6 C \operatorname{diam}(Q))) .
$$

Now, fix $\omega \in E, j \in \mathbf{Z}$ and write

$$
a_{j}=\#\left\{Q \in \mathbf{W}_{j}: \omega \in S_{E}(Q)\right\}=\#\left\{Q \in \mathbf{W}_{j}: Q \in P(\omega)\right\} .
$$

Noticing that the cubes $Q \in \mathbf{W}_{j}$ are essentially disjoint, we have

$$
a_{j} \cdot 2^{-j n}=C(n) \sum_{Q \in \mathbf{W}_{j} \cap P(\omega)}|Q| \leq C(n)|B(\omega, \varphi(6 C \operatorname{diam}(Q)))| \leq C(n) \varphi\left(6 C \cdot 2^{-j}\right)^{n} .
$$

Lemma 4.2 can be improved if we add more assumptions for the function $\varphi$. More precisely, we have the following result.

Lemma 4.3. Let $\Omega \subset \mathbf{R}^{n}$ be a $\varphi$-length John domain, and let $\mathbf{W}$ be a Whitney decomposition of $\Omega$. Let $E \subset \Omega$. If

$$
\varphi^{-1}(a \cdot b) \geq C \varphi^{-1}(a) \cdot \varphi^{-1}(b)
$$

and $\sum_{i=1}^{\infty} \frac{2^{i}}{\varphi^{-1}\left(2^{i}\right)}<\infty$, then

$$
\#\left\{Q \in \cup_{i=1}^{j} \mathbf{W}_{i}: \omega \in S_{E}(Q)\right\} \leq C \frac{\varphi\left(2^{-j}\right)}{2^{-j}} .
$$

Proof. If $\omega \in S_{E}(Q)$, then $Q \in P(\omega)$. Let $\gamma$ be a $\varphi$-John curve joining $\omega$ to $x_{0}$.

Let $r=2^{-j}$. Divide $\gamma$, starting from $\omega$, into pieces of length $\varphi(r), 2 \varphi(r), \ldots$, $2^{i} \varphi(r), \ldots$ This is a finite sequence since $\Omega$ is bounded. It is obvious that at most $C \frac{\varphi(r)}{r}$ Whitney cubes with diameter greater than $r$ intersect the first piece of $\gamma$. Measuring in terms of arc length, the piece corresponding $2^{i} \varphi(r)$ is at least

$$
\varphi(r)+2 \varphi(r)+\cdots+2^{i-1} \varphi(r) \approx 2^{i} \varphi(r)
$$

away from $\omega$, and so its distance to the boundary is at least

$$
\varphi^{-1}\left(2^{i} \varphi(r)\right) \gtrsim \varphi^{-1}\left(2^{i}\right) r
$$

by condition (1.2) for $\varphi$. Hence it is covered by no more than $C \frac{2^{i} \varphi(r)}{\varphi^{-1}\left(2^{i}\right) r}$ Whitney cubes that intersect $\gamma$. Thus the total number of Whitney cubes with diameter greater than $r$ does not exceed

$$
C\left(\frac{\varphi(r)}{r}+\sum_{i=1}^{\infty} \frac{2^{i} \varphi(r)}{\varphi^{-1}\left(2^{i}\right) r}\right) \lesssim \frac{\varphi(r)}{r} .
$$


Note that we have excluded the simplest John case, namely $\varphi(t) \approx t$, in Lemma 4.3. The reason is that Lemma 4.2 is already sharp in the John case. Of course, one can easily adapt the estimate from the proof of Lemma 4.3 as well.

Corollary 4.4. Under the assumptions of Lemma 4.3, we have that

$$
\sum_{Q \in \bigcup_{i=1}^{j} \mathbf{W}_{i}} \mu\left(S_{E}(Q)\right) \leq C \frac{\varphi\left(2^{-j}\right)}{2^{-j}} \mu(E)
$$

for any Borel measure $\mu$ on $\partial \Omega$.

Proof. Lemma 4.3 implies that

$$
\sum_{Q \in \bigcup_{i=1}^{j} \mathbf{W}_{i}} \mu\left(S_{E}(Q)\right)=\int_{E} \sum_{Q \in \bigcup_{i=1}^{j} \mathbf{W}_{i}} \chi_{S_{E}(Q)}(\omega) d \mu(\omega) \leq C \frac{\varphi\left(2^{-j}\right)}{2^{-j}} \mu(E) .
$$

\section{Growth of quasihyperbolic metric in $\varphi$-John domains}

In this section, we provide upper bounds for quasihyperbolic metric in $\varphi$-John domains.

Lemma 5.1. Let $\Omega \subset \mathbf{R}^{n}$ be a $\varphi$-length John domain with center $x_{0}$. Then there exists a positive constant $C$, depending only on the data, such that

$$
k_{\Omega}\left(x, x_{0}\right) \leq C \int_{d(x, \partial \Omega)}^{\varphi(C \operatorname{diam} \Omega)} \frac{1}{\varphi^{-1}(t)} d t+1 .
$$

Proof. Let $x \in \Omega$. We may connect $x$ and $x_{0}$ with a $\varphi$-length John curve $\gamma$. Suppose first that

$$
l\left(\gamma\left(x, x_{0}\right)\right) \leq \frac{1}{2} d(x, \partial \Omega)
$$

If $y \in \gamma\left(x, x_{0}\right)$, then

$$
d(y, \partial \Omega) \geq d(x, \partial \Omega)-l(\gamma(x, y)) \geq \frac{1}{2} d(x, \partial \Omega)
$$

and we obtain

$$
k_{\Omega}\left(x, x_{0}\right) \leq \int_{\gamma} \frac{d s}{d(y, \partial \Omega)} \leq 2 \int_{0}^{l\left(\gamma\left(x, x_{0}\right)\right)} \frac{d s}{d(x, \partial \Omega)} \leq 2 \frac{l\left(\gamma\left(x, x_{0}\right)\right)}{d(x, \partial \Omega)} \leq 1 .
$$

Next, we consider the case that

$$
l\left(\gamma\left(x, x_{0}\right)\right)>\frac{1}{2} d(x, \partial \Omega) .
$$

Choose a point $y_{0} \in \gamma$ such that

$$
l\left(\gamma\left(x, y_{0}\right)\right)=\frac{1}{2} d(x, \partial \Omega) .
$$

If $y \in \gamma\left(y_{0}, x_{0}\right)$, then

$$
d(y, \partial \Omega) \geq \frac{1}{C} \varphi^{-1}(l(\gamma(x, y)))
$$


and hence

$$
\begin{aligned}
k_{\Omega}\left(x, x_{0}\right) & \leq \int_{0}^{\frac{1}{2} d(x, \partial \Omega)} \frac{d s}{d(x, \partial \Omega)-s}+C \int_{\frac{1}{2} d(x, \partial \Omega)}^{l(\gamma)} \frac{d s}{\varphi^{-1}(s)} \\
& \leq \log 2+C \int_{d(x, \partial \Omega)}^{\varphi(C \operatorname{diam} \Omega)} \frac{d s}{\varphi^{-1}(s)} .
\end{aligned}
$$

An obvious modification of the above proof gives the following estimate.

Lemma 5.2. Let $\Omega \subset \mathbf{R}^{n}$ be a $\varphi$-length inner uniform domain. Then there exists a positive constant $C$, depending only on the data, such that

$$
k_{\Omega}(x, y) \leq C \int_{\min \{d(x, \partial \Omega), d(y, \partial \Omega)\}}^{C d_{I}(x, y)} \frac{1}{\varphi^{-1}(t)} d t+2
$$

for each pair $x, y$ of points in $\Omega$.

Lemma 5.3. Let $\Omega \subset \mathbf{R}^{n}$ be a $\delta$-Gromov-hyperbolic $\varphi$-dist John domain with center $x_{0}$. Then there exists a positive constant $C$, depending only on the data, such that

$$
k_{\Omega}\left(x, x_{0}\right) \leq C \int_{d(x, \partial \Omega)}^{C d_{I}\left(x, x_{0}\right)} \frac{t^{n-1}}{\left(\varphi^{-1}(t)\right)^{n}} d t .
$$

Proof. By Proposition 3.8, quasihyperbolic geodesics starting from the center in $\Omega$ are $\varphi$-dist John curves and note that the quasihyperbolic metric is comparable to the number of Whitney cubes that intersect the quasihyperbolic geodesic. The proof of [11, Lemma 3.7] applies with obvious modifications.

\section{Modulus of continuity of quasiconformal mappings between general domains}

We consider in this section the modulus of continuity of quasiconformal mappings between more general domains in $\mathbf{R}^{n}$.

Theorem 6.1. Let $\Omega^{\prime} \subset \mathbf{R}^{n}$ be a domain and $\Omega$ be a $\varphi$-length John domain. Suppose that there exist a constant $\lambda>0$ such that the function $g=\left(\varphi^{-1}\right)^{\lambda}$ is concave and a constant $\mu$ such that $\varphi(t) \leq C t^{\mu}$ for $t \in(0,1)$. Moreover, assume $p:=\frac{\lambda}{n-1}-1+\mu>0$. If $\varphi$ satisfies the assumptions in Lemma 4.3, then each quasiconformal mapping $f: \Omega^{\prime} \rightarrow \Omega$ satisfies

$$
D_{I}\left(f\left(x^{\prime}\right), f\left(y^{\prime}\right)\right) \leq C \Lambda^{-1}\left(\log \frac{1}{C d_{I}\left(x^{\prime}, y^{\prime}\right)}\right)^{1-n}
$$

where $\Lambda(t)=\left(\varphi^{-1}(t)\right)^{\lambda} t^{p(1-n)}$.

Remark 6.2. One can easily formulate the corresponding result for a $\varphi$-dist John domain using the estimates for the number of Whitney cubes in $\varphi$-dist John domains established in Section 4.

Let us consider our model $s$-John case in Theorem 6.1. In this case, $\varphi(t)=t^{1 / s}$ for $t \in(0,1)$ and we may choose $\lambda=\mu=\frac{1}{s}$. Then

$$
p>0 \text { if and only if } \frac{1}{s(n-1)}-1+\frac{1}{s}>0,
$$


which is further equivalent to

$$
s<1+\frac{1}{n-1} .
$$

Note also that the assumptions in Lemma 4.3 are fulfilled and hence we recover Theorem 1.2.

Theorem 6.3. Let $\Omega \subset \mathbf{R}^{n}$ be a $\varphi$-length John domain with diameter one satisfying all the assumptions of Theorem 6.1. Let $Q_{0}$ denote the Whitney cube containing the (John) center $x_{0}$. Then there exists a constant $C$ such that

$$
\operatorname{Cap}\left(E, Q_{0}, \Omega\right) \geq C \Lambda(\operatorname{diam} E)
$$

for all continua $E \subset \Omega$.

Proof. Let $\Omega \subset \mathbf{R}^{n}, n \geq 2$, be a $\varphi$-length John domain with diameter one and let $E \subset \Omega$ be a continuum. Let $u \in W^{1, n}(\Omega)$ be a test function for the $n$-capacity of the pair $\left(Q_{0}, E\right)$.

For each $x \in E$, we define a subpath $P^{\prime}(x)$ of $P(x)$ as follows: $P^{\prime}(x)=\left\{Q_{s}, \ldots\right.$, $\left.Q_{f}\right\}$ consists of a chain of Whitney cubes, which begins with the terminal cube $Q_{s}=Q(x)$ and continues back along the path $P(x)$ until it reaches the first cube $Q_{f}$ for which $\operatorname{diam}\left(Q_{f}\right) \geq \frac{1}{5} \operatorname{diam} E$. Since adjacent Whitney cubes $Q_{1}$ and $Q_{2}$ have $\operatorname{diam} Q_{1} \leq 5 \operatorname{diam} Q_{2}$, we must have $\operatorname{diam} Q \leq \operatorname{diam} E$ for all $Q \in P^{\prime}(x)$.

We claim that, without loss of generality, we can assume that $u_{Q(x)} \geq 2 / 3$ and $u_{Q_{f}} \leq 1 / 3$. First, suppose that $u_{Q(x)}<2 / 3$ for some $x \in E$. Then it follows from known results $[15$, Theorem 5.9] that

$$
\int_{\Omega}|\nabla u(x)|^{n} d x \geq \frac{1}{C}\left(\log \frac{1}{\operatorname{diam} E}\right)^{1-n} .
$$

Next suppose that the final cube $Q_{f}$ in the path $P^{\prime}(x)$ satisfies $u_{Q_{f}}>1 / 3$. Then we have two cubes $Q_{0}$ and $Q_{f}$ in the domain $\Omega$ and a continuous $W^{1, n}$-function $u$ satisfying $u=0$ on $Q_{0}$ and $u_{Q_{f}}>1 / 3$. In this case, we may invoke [17, Theorem 6.1] to deduce that

$$
\int_{\Omega}|\nabla u(x)|^{n} d x \geq \frac{1}{C}\left(\log \frac{1}{\operatorname{diam} Q_{f}}\right)^{1-n} \geq \frac{1}{C}\left(\log \frac{1}{\operatorname{diam} E}\right)^{1-n}
$$

since $\operatorname{diam} Q_{f} \geq \frac{1}{5} \operatorname{diam} E$.

Therefore, we may assume that $u_{Q(x)} \geq 2 / 3$ and $u_{Q_{f}} \leq 1 / 3$. In this situation, a straightforward chaining argument involving the Poincaré inequality on Whitney cubes along the path $P^{\prime}(x)$ yields the estimate

$$
1 \leq C \sum_{Q \in P^{\prime}(x)} \operatorname{diam} Q f_{Q}|\nabla u(y)| d y .
$$

Now choose a Frostman measure $\mu$ on the continuum $E$ with growth function $g(r)=\left(\varphi^{-1}(r)\right)^{\lambda}$, i.e. a Borel measure supported on $E$ satisfying

$$
\mu(E \cap B(x, r)) \leq g(r)
$$

for all balls $B(x, r)$ and

$$
\mu(E) \geq \frac{1}{C(n)} \mathcal{H}_{g}^{\infty}(E)
$$


Since $g$ is concave by assumptions, we further have

$$
\mu(E) \geq \frac{1}{C(n)} g(\operatorname{diam} E) .
$$

Integrating (6.3) over the set $E$ with respect to the Frostman measure $\mu$ and applying Hölder's inequality, we see that

$$
\mu(E) \leq C \int_{E} \sum_{Q \in P^{\prime}(x)}\left(\int_{Q}|\nabla u(y)|^{n} d y\right)^{1 / n} d \mu(x) .
$$

We now interchange the order of summation and integration to deduce that

$$
\mu(E) \leq C \sum_{Q \in \mathbf{W}, \operatorname{diam} Q \leq \operatorname{diam} E} \mu\left(S_{E}(Q)\right)\left(\int_{Q}|\nabla u(y)|^{n} d y\right)^{1 / n} .
$$

Applying Hölder's inequality again leads to

$$
\begin{aligned}
\mu(E) & \leq C\left(\sum_{Q \in \mathbf{W}, \operatorname{diam} Q \leq \operatorname{diam} E} \mu\left(S_{E}(Q)\right)^{n /(n-1)}\right)^{\frac{n-1}{n}}\left(\sum_{Q \in \mathbf{W}} \int_{Q}|\nabla u(y)|^{n} d y\right)^{1 / n} \\
& \leq C\left(\sum_{Q \in \mathbf{W}, \operatorname{diam} Q \leq \operatorname{diam} E} \mu\left(S_{E}(Q)\right)^{n /(n-1)}\right)^{\frac{n-1}{n}}\left(\int_{\Omega}|\nabla u(y)|^{n} d y\right)^{1 / n} .
\end{aligned}
$$

We require an estimate for terms of the form

$$
\sum_{Q \in \mathbf{W}, \operatorname{diam} Q \leq \operatorname{diam} E} \mu\left(S_{E}(Q)\right)^{1+\delta}
$$

with $\delta=\frac{1}{n-1}$, which we give in the following lemma.

Lemma 6.4. Let $\Omega$ be a $\varphi$-length John domain in $\mathbf{R}^{n}$ with diameter one. Suppose that $\mu$ is a Borel measure on $\mathbf{R}^{n}$ which satisfies the growth condition $\mu(B(x, r)) \leq g(r)$. Then there exists a constant $C$ such that

$$
\sum_{j=j_{0}}^{\infty} \sum_{Q \in \mathbf{W}_{j}} \mu\left(S_{E}(Q)\right)^{1+\delta} \leq C \mu(E) 2^{-j_{0} p}
$$

with $j_{0} \leq C \log (1 / \operatorname{diam} E)$ so that $\operatorname{diam} Q \leq \operatorname{diam} E$ implies that $Q \in \mathbf{W}_{j}$ for some $j \geq j_{0}$.

By Lemma 6.4 and (6.6),

$$
\mu(E)^{n} \leq C \mu(E)^{n-1} 2^{-j_{0} p(n-1)} \int_{\Omega}|\nabla u|^{n} .
$$

It follows that

$$
\int_{\Omega}|\nabla u|^{n} \geq C \mu(E) 2^{-j_{0} p(1-n)} \geq C\left(\varphi^{-1}(\operatorname{diam} E)\right)^{\lambda} \cdot(\operatorname{diam} E)^{p(1-n)} .
$$


Proof of Lemma 6.4. The growth condition on $\mu$ implies that

$$
\begin{aligned}
\sum_{Q \in \mathbf{W}, \operatorname{diam} Q \leq \operatorname{diam} E} \mu\left(S_{E}(Q)\right)^{1+\delta} & \leq \sum_{j=j_{0}}^{\infty} \sum_{Q \in \mathbf{W}_{j}} \mu\left(S_{E}(Q)\right) \mu\left(S_{E}(Q)\right)^{\delta} \\
& \leq \sum_{j=j_{0}}^{\infty} \sum_{Q \in \mathbf{W}_{j}} \mu\left(S_{E}(Q)\right) g\left(\operatorname{diam} S_{E}(Q)\right)^{\delta} \\
& \leq \sum_{j=j_{0}}^{\infty} \sum_{Q \in \mathbf{W}_{j}} \mu\left(S_{E}(Q)\right) g(C \varphi(\operatorname{diam} Q))^{\delta} \\
& \leq C \sum_{j=j_{0}}^{\infty} \sum_{Q \in \mathbf{W}_{j}}\left(2^{-j}\right)^{\lambda \delta} \mu\left(S_{E}(Q)\right) .
\end{aligned}
$$

Set $a_{j}=\sum_{Q \in \mathbf{W}_{j}} \mu\left(S_{E}(Q)\right)$ and let $A_{j}=a_{1}+\cdots+a_{j}$. We apply summation by parts to obtain

$$
\sum_{j=j_{0}}^{\infty}\left(2^{-j}\right)^{\lambda \delta} a_{j} \leq \sum_{j=j_{0}}^{\infty}\left(\left(2^{-j}\right)^{\lambda \delta}-\left(2^{-j-1}\right)^{\lambda \delta}\right) A_{j} .
$$

Corollary 4.4 implies that $A_{j} \leq C \frac{\varphi\left(2^{-j}\right)}{2^{-j}} \mu(E)$ for each $j$ and so

$$
\begin{aligned}
\sum_{Q \in \mathbf{W}, \operatorname{diam} Q \leq \operatorname{diam} E} \mu\left(S_{E}(Q)\right)^{1+\delta} & \leq C \mu(E) \sum_{j=j_{0}}^{\infty}\left(\left(2^{-j}\right)^{\lambda \delta}-\left(2^{-j-1}\right)^{\lambda \delta}\right) \frac{\varphi\left(2^{-j}\right)}{2^{-j}} \\
& \leq C \mu(E) \sum_{j=j_{0}}^{\infty} 2^{-j p} \leq C \mu(E) 2^{-j_{0} p}
\end{aligned}
$$

by our assumptions in Theorem 6.1.

Proof of Theorem 6.1. This follows directly from Theorem 6.3 and the arguments used in the beginning of the proof of Theorem 1.1 below; see also [16, Proof of Theorem 1.1].

\section{Proofs of the main results}

Proof of Theorem 1.1. Let $\Omega \subset \mathbf{R}^{n}$ be a bounded $\delta$-Gromov-hyperbolic $\varphi$ length John domain with center $x_{0}$. Since $\Omega$ is bounded, we may scale $\Omega$ to have diameter one. Let $\mathbf{W}(\Omega)$ be a Whitney decomposition of $\Omega$. Fix a Whitney cube $F=Q_{0} \in \mathbf{W}(\Omega)$ with center $x_{0}$ and let $F_{0}^{\prime}=f^{-1}(F)$. Since $F=Q_{0}$ is a Whitney cube, $\frac{3}{2} Q_{0} \subset \Omega$. Let $F^{\prime}=f^{-1}\left(\frac{3}{2} Q_{0}\right)$. By elementary properties of quasiconformal mappings, there exists $\tau=\tau(n, K)>0$ so that the set of points $x \in \mathbf{R}^{n}$ with $\operatorname{dist}\left(x, F_{0}^{\prime}\right) \leq \tau \operatorname{diam} F_{0}^{\prime}$ is contained in $F^{\prime}$.

Let $x, y \in \Omega^{\prime}$. Note that $f$ is automatically Hölder continuous in the internal metric as a map from the compact subset $F^{\prime} \subset \Omega$ with the Euclidean (hence also the internal) metric into $\Omega$; the Hölder data depends only on $n, K$ and $\operatorname{dist}\left(f^{-1}\left(x_{0}\right), \partial \Omega^{\prime}\right)$. Thus we may assume that either $x$ or $y$ is in $\Omega^{\prime} \backslash F^{\prime}$; without loss of generality let this be the case for $x$.

Next, note that if $d_{I}(x, y) \gtrsim \tau \operatorname{diam} F_{0}^{\prime}$, then

$$
\frac{d_{I}(f(x), f(y))}{d_{I}(x, y)^{\alpha}} \leq C \frac{M}{\left(\operatorname{diam} F_{0}^{\prime}\right)^{\alpha}} \lesssim 1
$$


for any choice of $\alpha$. Thus it suffices to verify (1.3) in the case $d_{I}(x, y) \leq \frac{1}{2} \tau \operatorname{diam} F_{0}^{\prime} \lesssim$ $\operatorname{dist}\left(x, F_{0}^{\prime}\right)$. Let $[f(x), f(y)]$ be the quasihyperbolic geodesic in $\Omega$ that connects $f(x)$ and $f(y)$. Write $E^{\prime}=f^{-1}([f(x), f(y)])$. Then $\operatorname{diam} E^{\prime} \lesssim d_{I}(x, y) \leq \frac{1}{2} \tau \operatorname{diam} F_{0}^{\prime}$. A simple calculation shows that diam $E^{\prime} \leq \frac{1}{2} \tau \operatorname{diam} F_{0}^{\prime}<\frac{1}{2} \operatorname{dist}\left(E^{\prime}, F_{0}^{\prime}\right)$. A fundamental property of the conformal capacity states that in this case

$$
\operatorname{Cap}\left(E^{\prime}, F_{0}^{\prime}, \Omega^{\prime}\right) \leq C\left(\log \frac{\operatorname{dist}\left(E^{\prime}, F_{0}^{\prime}\right)}{\operatorname{diam} E^{\prime}}\right)^{1-n} .
$$

Now we need to estimate $\operatorname{Cap}([f(x), f(y)], F, \Omega)$ from below. Let us consider the quasihyperbolic triangle

$$
\left[x_{0}, f(x)\right] \cup[f(x), f(y)] \cup\left[f(y), x_{0}\right] .
$$

Let $z$ be the midpoint of $[f(x), f(y)]$ with respect to arc length. Since $\Omega$ is $\delta$-Gromovhyperbolic, we find $\omega$ in the union of the other two sides of the triangle so that $k_{\Omega}(z, \omega) \leq M$ and $d_{I}(z, \omega) \leq M d(z, \partial \Omega)$, where we may assume that $M \geq 1$. Assume, without loss of generality that $\omega \in\left[f(y), x_{0}\right]$.

Suppose first that $d(z, \partial \Omega) \leq \frac{1}{4 a^{2} M} l([f(x), f(y)])$, where $a$ is the constant from the Gehring-Hayman inequality (3.2). Then an elementary computation shows that necessarily

$$
l([f(y), \omega]) \geq \frac{1}{4 a} l([f(x), f(y)]) .
$$

Using Proposition 3.8 and Lemma 5.1, we conclude that

$$
\begin{aligned}
k_{\Omega}\left(z, x_{0}\right) & \leq k_{\Omega}(z, \omega)+k_{\Omega}\left(\omega, x_{0}\right) \leq M+C \int_{l([f(x), f(y)) / 4 a}^{l\left(\left[f(y), x_{0}\right]\right.} \frac{d s}{\varphi^{-1}(s)}+1 \\
& \leq C^{\prime} \int_{l([f(x), f(y)) / 4 a}^{l\left(\left[f(y), x_{0}\right]\right.} \frac{d s}{\varphi^{-1}(s)},
\end{aligned}
$$

where the last inequality follows from the reductions in our first paragraph.

Suppose then that $d(z, \partial \Omega) \geq \frac{1}{4 a^{2} M} l([f(x), f(y)])$. Using Lemma 5.1 for $\left[z, x_{0}\right]$, we conclude that

$$
k_{\Omega}\left(z, x_{0}\right) \leq \int_{l([f(x), f(y)]) / 4 a^{2} M}^{l\left(\left[z, x_{0}\right]\right)} \frac{d s}{\varphi^{-1}(s)}+1 .
$$

Now the $\varphi$-length John assumption together with Proposition 3.8 implies that $l\left(\left[z, x_{0}\right]\right), l\left(\left[f(y), x_{0}\right] \leq M^{\prime}\right.$ for some $M^{\prime}<\infty$. Since $\varphi^{-1}$ is doubling, we thus conclude from the previous two paragraphs that

$$
k_{\Omega}\left(z, x_{0}\right) \leq C^{\prime \prime} \int_{l([f(x), f(y)])}^{M^{\prime}} \frac{d s}{\varphi^{-1}(s)} .
$$

Let $u$ be an admissible function for $\operatorname{Cap}([f(x), f(y)], F, \Omega)$. Let $Q_{z}$ be the Whitney cube that contains $z$ and we study two cases. First, we assume that $\left|u_{Q_{z}}\right|<\frac{2}{3}$. Then necessarily $\left|u_{F}-u_{Q_{z}}\right| \geq \frac{1}{3}>0$. It follows from a standard chaining argument involving the Poincaré inequality on Whitney cubes that

$$
\int_{\Omega}|\nabla u|^{n} d x \gtrsim k_{\Omega}\left(x_{0}, z\right)^{1-n}
$$


see e.g. [16, p. 28]. Next, we assume that $u_{Q_{z}} \geq \frac{2}{3}$. Note that $\left.u\right|_{E} \leq 0$, where $E=[f(x), f(y)] \cap Q_{z}$, and that $\operatorname{diam} E \geq \operatorname{diam} Q_{z}$, one easily deduces that

$$
\int_{\Omega}|\nabla u|^{n} d x \gtrsim 1
$$

see [17]. Therefore

$$
\operatorname{Cap}([f(x), f(y)], F, \Omega) \gtrsim\left(\int_{l([f(x), f(y)])}^{M^{\prime}} \frac{d s}{\varphi^{-1}(s)}\right)^{1-n} .
$$

The desired estimate in the $\varphi$-length John case follows by combining (7.2) and (7.3).

For the $\varphi$-dist John case we argue analogously, replacing length by distance in the definition of $z$. The only substantial change is that, in the estimate for $k_{\Omega}\left(z, x_{0}\right)$, we apply Lemma 5.3 to conclude that

$$
k_{\Omega}\left(z, x_{0}\right) \leq \tilde{C} \int_{l([f(x), f(y)])}^{M^{\prime}} \frac{s^{n-1} d s}{\left(\varphi^{-1}(s)\right)^{n}} .
$$

This gives the desired estimate by the above argument.

Proof of Theorem 1.2. The proof relies on the arguments contained in [12]. We present the details here for completeness.

Note that we only need a capacity estimate of the form as in Theorem 6.3, i.e. $\operatorname{Cap}\left(E, Q_{0}, \Omega\right)^{\frac{1}{(n-1)(s-1)}} \geq C \operatorname{diam} E$ for all continua $E \subset \Omega$. To this end, let $u \in C^{\infty}(\Omega)$ be a test function. Let $\mathbf{W}=\mathbf{W}(\Omega)$ be a Whitney decomposition of $\Omega$. Let $Q_{i}, i=1, \ldots, m$ be those Whitney cubes that intersect $E$. It is clear that

$$
\operatorname{diam} E \leq \sum_{i=1}^{m} \operatorname{diam} Q_{i}
$$

Fix one such Whitney cube $Q_{i_{0}}$ and let $x_{i_{0}}$ be its center. Fix $s \in\left(1,1+\frac{1}{n-1}\right)$ and $\Delta>0$ small so that $(s-1)(n-1)+\Delta \leq 1$. As before, we may assume that $u_{Q_{i_{0}}} \geq \frac{1}{2}$. Let $Q_{i_{0}}^{j}, j=1, \ldots, k$ be the Whitney cubes in $P\left(x_{i_{0}}\right)$ with $Q_{i_{0}}^{k}=Q_{i_{0}}$. The chaining argument involving Poincaré inequality gives us the similar estimate

$$
1 \lesssim \sum_{j=1}^{k} \operatorname{diam} Q_{i_{0}}^{j} f_{Q_{i_{0}}^{j}}|\nabla u(y)| d y
$$

Hölder's inequality implies

$$
1 \lesssim\left(\sum_{j=0}^{k} r_{j}^{(1-\kappa) n /(n-1)}\right)^{(n-1) / n}\left(\sum_{j=0}^{k} r_{j}^{\kappa n-n} \int_{Q_{i_{0}}^{j}}|\nabla u|^{n}\right)^{1 / n}
$$

where $r_{j}=\operatorname{diam} Q_{i_{0}}^{j}$ and $\kappa=\frac{s+n-1-\Delta}{s n}$. Using Lemma 4.3, one can easily conclude

$$
\sum_{j=0}^{k} r_{j}^{(1-\kappa) n /(n-1)}<C
$$


Indeed, the number of $r_{i}$ with $2^{-(j+1)} \leq r_{i} \leq 2^{-j}$ does not exceed $C 2^{j(s-1) / s}$, and hence

$$
\sum_{i=0}^{k} r_{i}^{(1-\kappa) n /(n-1)} \leq C \sum_{j=0}^{\infty} 2^{j(s-1) / s} 2^{-j(1-\kappa) n /(n-1)}=C \sum_{j=0}^{\infty} 2^{-j \frac{\Delta}{s(n-1)}}<C .
$$

Therefore,

$$
\sum_{j=0}^{k} r_{j}^{\kappa n-n} \int_{Q_{i_{0}}^{j}}|\nabla u|^{n} \geq C
$$

where the constant $C$ depends only on $n, \Delta$ and the constant from $s$-John condition.

By the $s$-John condition $C r_{j} \geq\left|x_{i_{0}}-y\right|^{s}$, for $y \in Q_{i_{0}}^{j}$, and since $\kappa n-n<0$ according to our choice, we obtain

$$
r_{j}^{\kappa n-n} \lesssim\left|x_{i_{0}}-y\right|^{s(\kappa n-n)}
$$

for $y \in Q_{i_{0}}^{j}$. For $y \in Q_{i_{0}}^{i} \cap\left(2^{j+1} Q_{i_{0}} \backslash 2^{j} Q_{i_{0}}\right)$, we have $\left|x_{i_{0}}-y\right| \approx 2^{j} r_{k}$ and hence for such $y$,

$$
r_{i}^{\kappa n-n} \lesssim\left(2^{j} r_{k}\right)^{s(\kappa n-n)}
$$

Combining (7.4) with (7.5) leads to

$$
\begin{aligned}
& 1 \lesssim \sum_{j=0}^{k} r_{j}^{\kappa n-n} \int_{Q_{i_{0}}^{j}}|\nabla u|^{n} \lesssim\left(r_{k}\right)^{s(\kappa n-n)} \int_{Q_{i_{0}}}|\nabla u|^{n} \\
& +\sum_{j=0}^{\left|\log r_{k}\right|}\left(2^{j} r_{k}\right)^{s(\kappa n-n)} \int_{\left(2^{j+1} Q_{i_{0}} \backslash 2^{j} Q_{i_{0}}\right) \cap \Omega}|\nabla u|^{n} \\
& \lesssim \sum_{l=0}^{\left|\log r_{k}\right|+1}\left(2^{l} r_{k}\right)^{s(\kappa n-n)} \int_{2^{l} Q_{i_{0}} \cap \Omega}|\nabla u|^{n} .
\end{aligned}
$$

On the other hand,

$$
\sum_{l=0}^{\left|\log r_{k}\right|+1}\left(2^{l} r_{k}\right)^{\Delta}<r_{k}^{\Delta} \sum_{l=-\infty}^{\left|\log r_{k}\right|+1} 2^{l \Delta}<C
$$

Comparing the above two estimates, we conclude that there exists an $l$ such that

$$
\left(2^{l} r_{k}\right)^{\Delta} \lesssim\left(2^{l} r_{k}\right)^{s(\kappa n-n)} \int_{2^{l} Q_{i_{0}} \cap \Omega}|\nabla u|^{n}
$$

It follows that,

$$
\int_{\Omega \cap 2^{l} Q_{i_{0}}}|\nabla u|^{n} \gtrsim\left(2^{l} r_{k}\right)^{s(n-\kappa n)+\Delta} .
$$

In other words, there exists an $R_{x} \geq d(x, \partial \Omega) / 2$ with

$$
\left(\int_{\Omega \cap B\left(x, R_{x}\right)}|\nabla u|^{n}\right)^{\frac{1}{(n-1)(s-1)+\Delta}} \gtrsim R_{x}
$$


Applying the Vitali covering lemma to the covering $\left\{B\left(x, R_{x}\right)\right\}_{x \in E}$ of the set $E$, we can select pairwise disjoint balls $B_{1}, \ldots, B_{k}, \ldots$ such that $E \subset \bigcup_{i=1}^{\infty} 5 B_{i}$. Let $r_{i}$ denote the radius of the ball $B_{i}$. Then

$$
\operatorname{diam} E \leq \sum_{i=1}^{\infty} \operatorname{diam} 5 B_{i}=5 \sum_{i=1}^{\infty} r_{i} \lesssim \sum_{i=1}^{\infty}\left(\int_{\Omega \cap B_{i}}|\nabla u|^{n}\right)^{\frac{1}{(n-1)(s-1)+\Delta}}
$$

The desired capacity estimate follows by noticing the elementary inequality

$$
\sum_{i} a_{i}^{p} \lesssim\left(\sum_{i} a_{i}\right)^{p}, \quad p \geq 1
$$

Acknowledgements. I wish to express my deep gratitude to my supervisor Academy Professor Pekka Koskela for many useful suggestions. I am also grateful to the referee whose helpful comments substantially improved the exposition.

\section{References}

[1] Becker, J., and Ch. Pommerenke: Hölder continuity of conformal maps with quasiconformal extension. - Complex Variables Theory Appl. 10:4, 1988, 267-272.

[2] Bonk, M., J. Heinonen, and P. Koskela: Uniformizing Gromov hyperbolic spaces. Astérisque 270, 2001.

[3] Gehring, F. W., and W. K. Hayman: An inequality in the theory of conformal mapping. J. Math. Pures Appl. (9) 41, 1962, 353-361.

[4] Gehring, F. W., and O. Martio: Lipschitz classes and quasiconformal mappings. - Ann. Acad. Sci. Fenn. Ser. A I Math. 10, 1985, 203-219.

[5] Gehring, F. W., and B. G. Osgood: Uniform domains and the quasihyperbolic metric. - J. Analyse Math. 36, 1979, 50-74.

[6] Gehring, F.W., and B. P. PAlkA: Quasiconformally homogeneous domains. - J. Analyse Math. 30, 1976, 172-199.

[7] Guo, C.-Y.: Generalized quasidisks and the associated John domains. - Report of Univ. of Jyväskylä, Dept. of Mathematics and Statistics, vol. 138, Univ. of Jyväskylä, Jyväskylä, 2013.

[8] Guo, C.-Y.: Generalized quasidisks and conformality II. - Proc. Amer. Math. Soc. (to appear).

[9] Guo, C.-Y., and P. Koskela: Generalized John disks. - Cent. Eur. J. Math. 12:2, 2014, 349-361.

[10] Guo, C.-Y., and P. Koskela: Sharpness of uniform continuity of quasiconformal mappings onto $s$-John domains. - Preprint, 2013.

[11] Guo, C.-Y., P. Koskela, and J. Takkinen: Generalized quasidisks and conformality. Publ. Mat. 58:1, 2014, 193-212.

[12] Hajlasz, P., and P. Koskela: Isoperimetric inequalities and imbedding theorems in irregular domains. - J. London Math. Soc. (2) 58:2, 1998, 425-450.

[13] Heinonen, J.: Quasiconformal mappings onto John domains. - Rev. Mat. Iberoamericana 5:3-4, 1989, 97-123.

[14] Heinonen, J., and S. Rohde: The Gehring-Hayman inequality for quasihyperbolic geodesics. - Math. Proc. Cambridge Philos. Soc. 114:3, 1993, 393-405.

[15] Heinonen, J., and P. Koskela: Quasiconformal maps in metric spaces with controlled geometry. - Acta Math. 181, 1998, 1-61. 
[16] Hencl, S., and P. Koskela: Quasihyperbolic boundary conditions and capacity: uniform continuity of quasiconformal mappings. - J. Anal. Math. 96, 2005, 19-35.

[17] Herron, D., and P. Koskela: Conformal capacity and the quasihyperbolic metric. - Indiana Univ. Math. J. 45:2, 1996, 333-359.

[18] John, F.: Rotation and strain. - Comm. Pure Appl. Math. 14, 1961, 391-413.

[19] Koskela, P., and T. Nieminen: Uniform continuity of quasiconformal mappings and conformal deformations. - Conform. Geom. Dyn. 12, 2008, 10-17.

[20] Koskela, P., J. Onninen, and J. T. Tyson: Quasihyperbolic boundary conditions and capacity: Hölder continuity of quasiconformal mappings. - Comment. Math. Helv. 76:3, 2001, 416-435.

[21] Martio, O., and J. Sarvas: Injectivity theorems in plane and space. - AnnÁcad. Sci. Fenn. Ser. A I Math. 4:2, 1979, 383-401.

[22] NÄKKI, R., and J. VÄIsÄLÄ: John disks. - Expo. Math. 9:1, 1991, 3-43.

[23] Pommerenke, Ch.: Boundary behaviour of conformal maps. - Grundlehren Math. Wiss. 299, Springer-Verlag, Berlin, 1992.

[24] Smith, W., and D. A. Stegenga: Hölder and Poincaré domains. - Trans. Amer. Math. Soc. 319, 1990, 67-100.

Received 13 August 2013 • Accepted 29 July 2014 